\section{Die ausführliche Rezension}

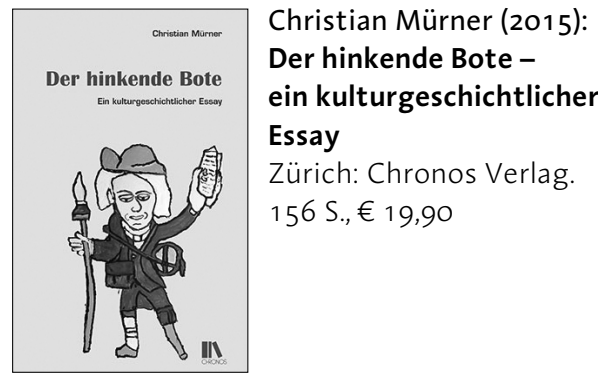

Dieses Buch ist weit weniger harmlos, als es der Aufmachung und vor allem auch dem Umfang nach den Anschein macht. Christian Mürner wirft darin eine Frage auf, die eigentlich schon seit beinahe drei Jahrhunderten auf der Hand liegen würde, die aber bislang noch von niemandem in dieser Form gestellt worden ist: Wie kommt es, dass die überaus populären und in vielen Regionen verbreiteten Jahreskalender, ein über lange Zeit dominierendes Informationsmedium, eine Symbolfigur zu ihrem berühmt gewordenen Emblem erheben, die eigentlich eine Randfigur der Gesellschaft ist und die im Übrigen kaum etwas mit dem tatsächlichen Inhalt zu tun hat?

Diese fast schon kuriose Frage verliert schnell an ihrer vermeintlich selbstvergessenen Detailversessenheit, wenn man ihr unterstellt, dass sie ein durchaus geeignetes Versuchsfeld für die Bearbeitung eines tiefgreifenden und frappierenden Widerspruchs bietet, der sich beinahe bruchlos durch unsere gesamte Geschichte zieht: Wie kommt es, dass nahezu alle Gesellschaften zu nahezu allen Zeiten das Thema Behinderung philosophisch, juristisch, literarisch und künstlerisch so auffällig prominent behandeln, obschon sich die Träger einer Behinderung selbst zumeist an die Ränder der Gesellschaft gestellt sehen? Wie kommt es zu diesem unserem eigentümlichen Verhältnis zum Thema Behinderung?

Mürner begnügt sich nicht mit den Abkürzungen sozialpsychologischer Theorien, die diesen Umstand mit griffigen Modellen erklären wollen, sondern er begibt sich auf eine ausgiebige Suche in den literarischen und künstlerischen Hinterlassenschaften vergangener Zeiten. Mit beneidenswert geduldiger Akribie trägt er prägnante Details zusammen, die sich um die immer etwas ominös bleibende Gestalt des hinkenden Boten anhäufen. Er befragt Zeitzeugen, versichert sich aber auch bei heutigen Autoren. Er betrachtet unterschiedliche Darstellungen und ordnet sie in modernen kulturgeschichtlichen Deutungen wie der sogenannten visuellen Wende, der iconic turn, wieder neu. Dazwischen streut Mürner mit der ihm eigenen rhetorischen Leidenschaft Anekdoten, welche die Leser zum Schmunzeln anregen und die sie dennoch so unnachahmlich tief in ihrer eigenen Unsicherheit treffen. Wussten sie beispielsweise, dass sich Charlie Chaplin in Malibu einen riesigen Spaß daraus gemacht hat, den völlig verdatterten Theodor W. Adorno nachzuäffen, als dieser geistesabwesend die dargebotene eiserne Prothese eines Schauspielers schüttelte, der seine Hand im Krieg verloren hatte? Adorno soll sich zunächst sehr erschrocken haben, um danach geistesgegenwärtig eine verbindliche Grimasse aufzusetzen, was nur umso peinlicher wirkte (S. 119). Vielleicht hätte der bei vielen unpassenden Gelegenheiten zitierte Knigge hier Rat gewusst (121)? Diese geradezu köstliche Entlarvung der fachlich wohl als irgendwie retardiert zu bezeichnenden Schwächen der Nichtbehinderten, und dies noch obendrein am personifizierten Beispiel des nachkriegsgeschichtlichen Gewissens Deutschlands, zeigt exemplarisch, dass es in Mürners Buch nicht nur um die Behinderten geht. Es geht um uns selbst. Streckenweise viel mehr, als uns lieb ist.

Mürner greift in seiner Argumentationslinie immer wieder in die offensichtlich reichen Schätze seiner literarischen Erfahrung. Aus vielen uns allen mehr oder minder bekannten Autorinnen und Autoren formt Mürner geradezu eine Klaviatur, die er für sein Essay und für seine Leserschaft bespielt. Nacheinander lässt er zum Beispiel Wilhelm und Jacob Grimm, Michel de Montaigne, Adam Smith, Georg Christoph Lichtenberg, Max Horkheimer, Arthur Schopenhauer, Hannah Arendt, John Dewey, Jürgen Habermas, Michel Foucault, Niklas Luhmann, Erving Goffman, Rainer Maria Rilke, Adolph Freiherr von Knigge, Katharina Rutschky, Judith Butler, Zygmunt Bauman, Alexander Kluge, Umberto Eco, aber auch Heinz Bach, Wolfgang Jantzen, Günther Cloerkes, Markus Dederich, Gudrun Wansing und Franz Christoph für uns erklingen. Das Ergebnis sind 33 kurze Kapitel, die sich in teils unterhaltsame, teils aber auch unter einigen Anstrengungen zu lesende Abschnitte gliedern. 
Unter ihnen finden sich zum Beispiel Die Ästhetik der Behinderung oder Die Aufdringlichkeit des Normalen oder der Prothesengott oder die Erpressung derAufklärung. Manchmal schweift der Autor von seinen Zielen ab, aber ohne seinen Weg gänzlich zu verlassen. Bisweilen wird er redundant, aber ohne dass es ermüdend wirkt. Nicht immer lassen seine Kapitelüberschriften das erwarten, was ihnen tatsächlich folgt. Aber dafür stößt man auf gut platzierte Geschichten und überraschende Wendungen. Man hat sich nachweislich schon vor 150 Jahren ausführlich Gedanken über die Anmut des Hässlichen gemacht. Im Hôtel des Invalides in Paris waren im ausgehenden 18. Jahrhundert fast tausend Personen untergebracht. Aber nur 22 von ihnen wiesen Amputationen auf und davon lediglich vier am Bein (S. 110). Und trotzdem hat es das Bild des hölzernen Beinersatzes in unser Kulturerbe geschafft. Jedes Kind vermeint sein rhythmisches, stumpfes Klopfen auf dem ebenfalls hölzernen Boden förmlich hören zu können. Altbekannt. Auch wenn es noch nie ein solches selbst gesehen haben mag. So ergeht es dem Leser des „Hinkenden Boten“. Er kann das Bild in Sekundenschnelle deuten. Auch wenn er, der aus der Zahl des Hôtel des Invalides abgeleiteten Wahrscheinlichkeit nach, noch nie einen tatsächlich Beinamputierten zu Gesicht bekommen hat. Die interpretierte Geschichte hat sich in die Köpfe gesaugt. Beinahe unentrinnbar.

Was genau nährt letztlich unser eigenes Verständnis von Behinderung? Wissenschaftliche Definitionen, nüchterne Fakten, einzelne Personen, persönlich erlebte oder erzählt bekommene Geschichten? Unterkomplexe, simplifizierende Bilder, deren Herkunft wir nur mit Mürners mühsam erforschten Daten zurückverfolgen können?

Der Autor argwöhnt, dass das Emblem des hinkenden Boten seiner Langsamkeit geschuldet ist. Immerhin tritt dagegen der eilige Bote auf seinem schnellen Pferd in einigen zeichnerischen Darstellungen in Erscheinung, wenn auch perspektivisch in den Hintergrund gerückt. Mit zahlreichen Zitaten als Stütze deklariert Mürner deshalb die Langsamkeit als Indiz für die Zuverlässigkeit der Nachricht. Ein abwegiger Gedanke in unserer doch so neuen, der Schnelligkeit verpflichteten Zeit. Und noch viel abwegiger, dass offenkundig schon unsere Vorfahren so gedacht haben könnten.
Mürners Antwort auf die Frage, weshalb wir uns derart viele Gedanken um Behinderung machen und ihre Träger dennoch nicht in unsere Mitte holen, ist keine abschließende. Vielmehr bietet er seiner Leserschaft eine kaleidoskopisch geformte Möglichkeit an.

Der Autor ist selbst nicht der eilige Bote, hoch zu Ross, der die schnellen Antworten liefern kann. Er ruft, seiner Hauptfigur gleich, zur Geduld auf. Geduld auf der Suche nach lohnenswerten Möglichkeiten. Mühsam hinkend nach Antworten sondierend. Machen Sie sich auf den Weg. Christian Mürner bietet sich Ihnen als nützliche Krücke an.

\section{Prof. Dr. Winfried Kronig}

$\mathrm{CH}-1700$ Freiburg

DOI 10.2378/vhn2016.art31d 